\title{
Population-based mathematical modeling antihypertensive drugs effect using BioUML platform
}

\author{
I.N. Kiselev ${ }^{1,2 *}$, A.F. Kolpakova ${ }^{1,2}$, F.A. Kolpakov ${ }^{1,2}$ \\ ${ }^{1}$ Institute of Computational Technologies, SB RAS, Novosibirsk \\ ${ }^{2}$ LLC «BIOSOFT.RU», Novosibirsk \\ *e-mail:axec@dote.ru
}

Key words: mathematical model, population modeling, cardiovascular system, arterial hypertension, antihypertensive drugs, BioUML

Motivation and Aim: Mathematical modeling can be used to predict effect of antihypertensive and other drugs on the modeling organism. Experimental data for drugs is usually given as population data (mean $\pm \mathrm{SD}$ ) thus in order to reproduce this data we need to transit from single mathematical models to populations of such models.

Methods and Algorithm: Population approach implies generating set of models reflecting real population of modeled organisms. Each particular model of this set represents distinct organism with certain parameters. We use metropolis algorithm to generate population of models with given distribution of observed values (systolic and diastolic blood pressure). When population is generated we extend the model by adding blocks describing pharmacokinetics and pharmacodynamics of selected drugs to each virtual patient thus describing treatment course (which can be monotherapy or therapy with several drugs) of given population. Finally we can compare results of treatment course for virtual and real populations.

Results: As a structural model we used mathematical model of the human cardiovascular system created in the BioUML platform and comprising modules (blocks) of different subsystems (heart, arterial system, salt-water balance, renin-aldosterone-angiotensin system and others). Using this model we have generated virtual population of nearly 1000 virtual organisms imitating patients with arterial hypertension (systolic pressure $148 \pm 15.5 \mathrm{mmHg}$, diastolic pressure $89 \pm 10 \mathrm{mmHg}$ ). Additionally to core model we have implemented detailed models of pharmacokinetics and pharmacodynamics of aliskiren [1] and losartan [2] and pharmacodynamics blocks of bisoprolol, enalapril and amlodipine. Created modules were used to simulate 8 different treatment courses for virtual population using said drugs including 4 different daily doses of aliskiren $(37.5 \mathrm{mg}$, $75 \mathrm{mg}, 150 \mathrm{mg}$ and $300 \mathrm{mg}$ ), daily dose of $100 \mathrm{mg}$ losartan [3], $5 \mathrm{mg}$ of amlodipine [4] and $5 \mathrm{mg}$ of enalapril [5], all courses have duration of 4 weeks. Separately treatment with biosprolol was tested in combination with physical stress [6]. Changes in blood pressure under different drugs were compared to results of published clinical studies and showed good agreement with experimental data except for diastolic pressure under effect of bisoprolol drug which indicates needed improvements in the model.

Conclusion: Implemented models and software provides effective platform for reproducing results of clinical trials.

Acknowledgements: Supported by RFBR, research project No. 16-01-00779 A.

\section{References}

1. Hong Y., Dingemanse J., Mager D.E. (2008) Pharmacokinetic/Pharmacodynamics Modeling of Renin Biomarkers in Subjects Treated With the Renin Inhibitor Aliskiren. Clinical Pharmacology Therapeutics. 84(1):136-43.

2. Nguyen H.Q., Lin J., Kimoto E., Callegari E., Tse S., Obach R.S. (2017) Prediction of Losartan-Active Carboxylic Acid Metabolite Exposure Following Losartan Administration Using Static and Physiologically Based Pharmacokinetic Models. J Pharm Sci. 106(9):2758-2777.

3. Stanton A., Jensen C., Nussberger K., O’Brien E. (2003) Blood pressure lowering in essential hypertension with an oral renin inhibitor, aliskiren. Hypertension. 42(6):1137-1143.

4. Guo C., Pei Q., Tan H., Huang Z., Yuan H., Yang G. (2015) Effects of genetic factors on the pharmacokinetics and pharmacodynamics of amlodipine in primary hypertensive patients. Biomedical reports. 2:195-200.

5. Ruddy M.C., Mroczek W.J. (1993) Comparison of Ramipril and Enalapril in Patients With Essential Hypertension. Pharmacotherapy. 13(3):224-228

6. Leopold G., Ungethum W., Pabst J., Simane Z., Buhring K.U., Wiemann H. (1986) Pharmacodynamic profile of bisoprolol, a new beta1-selective adrenoceptor antagonist. Br. J. clin. Pharmac 22:293-300. 